\title{
ORIGINAL ARTICLE Retrospective study of rehabilitation outcomes following spinal cord injury due to tumour
}

\author{
$\mathrm{M} \operatorname{Tan}^{1}$ and PW New ${ }^{2,3}$
}

Study design: Retrospective study. Objectives: To examine the rehabilitation outcomes for patients with spinal cord injury (SCI) due to tumour, with a focus on the impact of pain on disability and length of stay (LOS).

Setting: Public hospital inpatient spinal rehabilitation unit, Melbourne, Australia.

Methods: Retrospective open-cohort case series of consecutive patients admitted between 1/7/1996 and 30/6/2008 with a diagnosis of recent onset tumour causing $\mathrm{SCl}$.

Results: In all 108 patients were admitted, of whom $62 \%$ ( $n=67)$ were male. The median age was 61.5 years (interquartile range (IQR) 53.6-74). Most patients had paraplegia ( $n=97,89.8 \%)$ and a metastatic tumour $(n=71,65.7 \%)$ causing their SCI. For patients with a primary tumour the Rasch motor functional independence measure (FIM) gain between admission and discharge (median 17 , IQR 4-23) was significantly greater $(P=0.006)$ than for those with secondary tumour (median 7 , IQR from -4 to 15$)$. Pain was present in $52 \%$ of patients and had a significant adverse influence on the rehabilitation process, with FIM motor efficiencies of 0.09 in patients with documented pain compared with 0.39 in patients without pain $(P=0.01)$. Patients with pain had significantly prolonged median LOS of 56 days (IQR 32.5-84.5) compared with 42 days (IQR 20.5-72.5, $P=0.03$ ) without pain.

Conclusion: Patients with SCI due to tumour have the potential to benefit from a focused, specialised SCI rehabilitation programme to optimise their outcomes. Careful patient selection, modifying the focus of team goals and a close relationship with treating oncologists and palliative care teams is essential.

Spinal Cord (2012) 50, 127-131; doi:10.1038/sc.2011.103; published online 11 October 2011

Keywords: spinal neoplasms; pain; non-traumatic spinal cord injury; rehabilitation; rehabilitation outcome; recovery of function

\section{INTRODUCTION}

The dual impairments of having a spinal cord injury (SCI) resulting from a tumour can often result in devastating consequences for both patients and their families. Each condition has separate implications for symptom management, treatment, rehabilitation and prognosis.

Metastatic spinal cord compression occurs in about $5 \%$ of all cancer patients, usually resulting in paraplegia. ${ }^{1,2}$ The incidence of metastatic spinal cord compression varies by primary disease site and age. ${ }^{3}$ Typically, patients with metastatic SCI have primary tumours of the prostate, breast or lung. Primary spinal cord tumours are rare, accounting for $4-8 \%$ of central nervous system tumours and an incidence rate of 0.74 per $100000 .^{4}$

SCI resulting from tumours account for between 10 and $26 \%$ of admissions to spinal rehabilitation units. ${ }^{5,6}$ Previous reports on their rehabilitation outcomes have been published. ${ }^{7-10}$ These patients can have a relatively short survival, ${ }^{11,12}$ and have a peak age at the time of diagnosis of between 50 and 70 years. ${ }^{13}$ With the ageing population and improvements in early cancer diagnosis and treatment leading to increased survival, the number of patients with SCI due to tumour with the potential to benefit from rehabilitation is increasing.

Pain is a well-recognised complication in patients with cancer, including those with spinal epidural metastasis. ${ }^{14}$ However, the impact of pain on functional gains in rehabilitation has not been previously reported. This retrospective case series was planned to examine the rehabilitation outcomes for patients with SCI due to tumour, with a focus on the impact of pain on functioning and length of stay (LOS).

\section{METHODS}

The Spinal Rehabilitation Unit at Caulfield Hospital, Victoria, Australia is a 10-bed inpatient unit located in a public hospital. It is funded by the State health department and is one of two dedicated SCI rehabilitation units in the State. The unit focuses on rehabilitation for patients with non-traumatic SCI. Patients are referred from both private and public acute care hospitals. It is a routine practice for the rehabilitation physician or trainee in rehabilitation medicine attached to the unit to assess patients referred by acute hospitals to determine suitability for admission. The assessment process excludes those who are perceived to have a prognosis of less than 3 months. These patients are directed to palliative care services.

Patients are generally considered ready for discharge when all inpatient goals are achieved to allow a safe transfer of care to the ensuing destination. These goals generally include optimising the patient's functional status (especially continence), carer needs and essential equipment, and environmental access provisions. No third party has influence over the admission duration.

\section{Case identification}

The health information department at Caulfield Hospital used the International Classification of Diseases and Related Health Problems, 10th edition, Australian modification ${ }^{15}$ coding to identify patients with tumour causing their SCI who were discharged between 1/7/1996 and 30/6/2008. The medical files of potential cases were reviewed by one of the authors (MT) to confirm that

${ }^{1}$ Registrar, Spinal Rehabilitation Unit, Caulfield Hospital, Alfred Health, Caulfield, Victoria, Australia; ${ }^{2}$ Head, Spinal Rehabilitation Unit, Caulfield Hospital, Alfred Health, Caulfield, Victoria, Australia and ${ }^{3}$ Epworth-Monash Rehabilitation Medicine Unit, Monash University, Melbourne, Victoria, Australia

Correspondence: Dr M Tan, Department of Medicine, St Vincent's Hospital Melbourne, PO Box 2900, Fitzroy, Victoria 3065, Australia.

E-mail: michael.tan@svhm.org.au

Received 7 April 2011; revised and accepted 1 August 2011; published online 11 October 2011 
patients met the inclusion criteria. Only those who had a SCI due to a primary or secondary tumour were included. Patients were excluded if they had a chronic SCI or were re-admitted for late complications.

There were 116 patients that fulfilled the study criteria. Of these, eight medical records were unable to be located (five records had been destroyed as part of a previous hospital policy and three records were missing). We have recently published the demographic characteristics, tumour aetiologies, treatments and survival analysis of this cohort. ${ }^{12}$

\section{Data collection}

The medical records of eligible patients were reviewed by one of the authors (MT) and pertinent details extracted. These included the following: demographic information (date of birth and gender); tumour diagnosis and type (primary or secondary); dates of admission to the Spinal Rehabilitation Unit and subsequent discharge. As the exact neurological level of injury was not always documented, patients were grouped into either tetraplegia (C1-T1) or paraplegia (T2-S5). The American Spinal Injury Association (ASIA) Impairment Scale grades ${ }^{16}$ were dichotomised into complete (ASIA A, B and C) and incomplete (ASIA D).

\section{Functional independence measure (FIM) scores}

The FIM is the most commonly used functional measure of disability and rehabilitation outcome. ${ }^{17}$ It has separate motor and cognitive subscales, ${ }^{18}$ with the cognitive subscale having a very high-ceiling effect in patients with SCI, including non-traumatic SCI, so is of limited utility. ${ }^{19}$ Therefore, only the FIM motor subscale was recorded within $72 \mathrm{~h}$ of admission and discharge by the treating team, who were credentialed in its use. The raw FIM motor subscale scores were converted by Rasch analysis tables ${ }^{20}$ to a linear score on a 100-point scale with equal intervals to facilitate parametric analysis.

\section{Pain}

The presence of pain was recorded if documented in the patient notes along with evidence of administration and titration of analgesia in the medication charts. Allied health and multidisciplinary team meeting notes were examined for references to pain interfering with participation in therapy. Pain was categorised as either neuropathic, nociceptive or both according to the file notes. The broad categories of analgesic medication administered were also recorded. These included simple analgesics, opiates, non-steroidal anti-inflammatory drugs, anti-epileptic agents, tricyclic antidepressants, tramadol and intrathecal pump.

Information collected was de-identified and recorded electronically into a password-protected database. Database entry was performed using FileMaker Pro software (version 10.0; FileMaker, Inc., Clara, CA, USA). Following completion of data collection, a random sample of $10 \%$ of medical files were rechecked and compared with the database for errors. If an error rate greater than $5 \%$ was found, the study protocol required rechecking another random sample of $40 \%$ of patient records and correcting any errors identified.

\section{Data analysis}

Statistical analysis was performed using Stata software (version 9; Statacorp, College Station, TX, USA). Descriptive statistics were calculated for demographic characteristics and clinical features. Categorical variables were analysed using the $\chi^{2}$-test, with the Fisher exact method used where appropriate. Continuous variables were tested for normality with the Shapiro-Wilk $W$ test. Continuous variables were described as medians (interquartile range (IQR)). The two-sample Wilcoxon rank-sum (Mann-Whitney) test and Kruskal-Wallis test for equality of populations was used for non-parametric data.

Testing of the Rasch FIM motor discharge scores revealed a skewed distribution that was not normally distributed. The FIM motor gain was calculated by subtracting the discharge FIM motor score from the admission score. The FIM motor efficiency was calculated by dividing the change in raw FIM motor subscale scores by the LOS. The Wilcoxon signed-rank test was used to measure the significance of the gain in raw FIM motor scores for the primary and secondary tumour groups. The difference in raw FIM motor efficiency between primary and secondary tumour groups was tested with the two-sample Wilcoxon rank-sum test.

The effect of pain on the FIM motor efficiency and LOS were evaluated using the two-sample Wilcoxon rank-sum test. The raw discharge motor FIM was stratified by tumour type and the presence of pain issues, with effect of pain on each tumour group being evaluated using the two-sample Wilcoxon rank-sum test.

Statistical significance was defined as a $P<0.05$. Approval for this project was obtained from the Research and Ethics Unit at Alfred Health.

\section{RESULTS}

There were 108 patients discharged during the study period. The median age was 61.5 years (IQR 53.5-74, range 21-90). Most patients were males $62 \%(n=67)$. Paraplegia was much more common $(n=97$, $89.8 \%)$ than tetraplegia $9.3 \%(n=10 ; n=1,1 \%$ level not listed). There were $37 \%(n=40)$ of patients with incomplete SCI, and $44 \%(n=48)$ had complete SCI. The American Spinal Injury Association Impairment Scale levels were not recorded in 20 subjects.

Most cases had a metastatic disease causing their SCI $(n=71$, $65.7 \%)$ and a primary tumour was causative in $34.3 \%(n=37)$. A detailed breakdown of the specific diagnoses has been reported previously. ${ }^{12}$

\section{LOS and discharge destination}

The median LOS was 47.5 days (IQR 25-80.5, range 4-237). The majority $(n=67,62 \%)$ of patients returned to their private residence, $11 \%(n=12)$ required transfer to an acute hospital and did not return to rehabilitation, $10 \%(n=11)$ were discharged to a nursing home, $4.6 \%(n=5)$ were discharged to an assisted living residence (for example, hostel or supportive accommodation), 1.9\% $(n=2)$ died and $10.2 \%(n=11)$ were transferred for palliative care. In all $75 \%$ $(n=30)$ of incomplete SCI and $50 \%(n=24)$ of complete SCI patients returned home (Table 1). There was a trend for complete SCI patients to be discharged to palliative care, however, this failed to reach significance $(P=0.1)$.

\section{FIM scores}

FIM scores were missing from 21 hospital files and these patients were excluded from any analysis involving FIM. Patients who died during their inpatient stay were excluded from the analysis of FIM change. Admission and discharge FIM motor scores are shown in Table 2. Primary tumour patients had significantly better $(P=0.006)$ functional improvement, with a median Rasch FIM motor gain of 17 (IQR 4-23) when compared with secondary tumour patients, who had a median gain of 8 (IQR from -4 to 15 ).

As expected, the incomplete SCI group demonstrated significantly $(P=0.03)$ greater functional improvement, with a median Rasch FIM motor gain of 12 (IQR 5-18), compared with the complete SCI group, who had a median Rasch FIM motor change of only 4 (IQR 0-16).

The primary tumour group demonstrated a significantly better $(P=0.04)$ FIM motor efficiency of 0.33 (IQR $0.10-0.68$ ) compared

\section{Table 1 Discharge destination}

\begin{tabular}{lrrrrr}
\hline & \multicolumn{2}{c}{ Incomplete } & & \multicolumn{2}{c}{ Complete } \\
\cline { 2 - 3 } \cline { 5 - 6 } & $n$ & & & $n$ & $\%$ \\
\hline Private residence & 30 & 75.0 & & 24 & 50.0 \\
Hospital & 3 & 7.5 & & 8 & 16.7 \\
Nursing home & 4 & 10.0 & & 6 & 12.5 \\
Assisted living residence & 2 & 5.0 & & 1 & 2.1 \\
Deceased & 0 & 0.0 & & 2 & 4.1 \\
Palliative care & 1 & 2.5 & & 7 & 14.6 \\
Total & 40 & 100.0 & & 48 & 100.0 \\
\hline
\end{tabular}


Table 2 Admission and discharge motor FIM subscales

\begin{tabular}{|c|c|c|c|c|}
\hline & \multicolumn{2}{|l|}{ Primary } & \multicolumn{2}{|c|}{ Secondary } \\
\hline & Raw FIM & RASCH FIM & Raw FIM & RASCH FIM \\
\hline Admission motor & 34 (26 to 47,13 to 81 ) & 38 (33 to 45,0 to 67 ) & 31 ( 24 to 47,16 to 75$)$ & 37 (31 to 45,20 to 62 ) \\
\hline Discharge motor & 72 (37 to 81,14 to 86$)$ & $60(40$ to 67,10 to 74$)$ & 55 (24 to 73,13 to 86 ) & 50 (29 to 60,0 to 74$)$ \\
\hline FIM gain & $24(5$ to $36,-3$ to 53$)$ & 17 (4 to $23,-12$ to 32 ) & $10(-2$ to $24,-55$ to 48$)$ & $8(-4$ to $15,-57$ to 32$)$ \\
\hline \multirow[t]{3}{*}{ FIM efficiency } & $0.33(0.10$ to $0.68,-0.18$ to 1.41$)$ & & $0.16(-0.10$ to $0.45,-6.11$ to 4.75$)$ & \\
\hline & \multicolumn{2}{|c|}{ Incomplete } & \multicolumn{2}{|c|}{ Complete } \\
\hline & Raw FIM & RASCH FIM & Raw FIM & RASCH FIM \\
\hline Admission motor & 47 (29 to 62,21 to 81$)$ & 45 (35 to 53,28 to 67 ) & 26 (22 to 35,13 to 75$)$ & 33 (29 to 39,0 to 62 ) \\
\hline Discharge motor & 73 (55 to 81,13 to 86$)$ & $60(50$ to 67,0 to 74$)$ & 35 (22 to 58,13 to 86 ) & 39 (29 to 51,0 to 74$)$ \\
\hline FIM gain & 18 (7 to $28,-55$ to 53$)$ & 12 (5 to $18,-57$ to 32 ) & $5(0$ to $22,-13$ to 52$)$ & $4(0$ to $16,-33$ to 31$)$ \\
\hline FIM efficiency & $0.33(0.14$ to $0.65,-6.11$ to 1.27$)$ & & $0.08(0.0$ to $0.33,-0.68$ to 0.75$)$ & \\
\hline
\end{tabular}

Abbreviations: FIM, Functional independent measure; IQR, interquartile range.

All values listed as median (IQR, range). Deceased patients excluded.

Table 3 Pain

\begin{tabular}{|c|c|c|c|c|c|c|}
\hline & \multirow[t]{2}{*}{$\mathrm{n}$} & \multirow[t]{2}{*}{$\%$} & \multicolumn{2}{|c|}{ Tumor type } & \multicolumn{2}{|c|}{$\mathrm{SCl}$} \\
\hline & & & Primary & Secondary & Incomplete & Complete \\
\hline $\begin{array}{l}\text { Pain issues documented } \\
\text { during admission }\end{array}$ & 56 & 51.9 & 19 & 37 & 16 & 31 \\
\hline $\begin{array}{l}\text { Pain adverse impact } \\
\text { on rehabilitation }\end{array}$ & 23 & 21.3 & 6 & 17 & 7 & 13 \\
\hline $\begin{array}{l}\text { Nociceptive pain } \\
\text { impact on rehabilitation }\end{array}$ & 15 & 13.9 & 2 & 13 & 5 & 7 \\
\hline $\begin{array}{l}\text { Neuropathic pain } \\
\text { impact on rehabilitation }\end{array}$ & 7 & 6.5 & 3 & 4 & 2 & 5 \\
\hline \multicolumn{7}{|l|}{ Pain type } \\
\hline Nociceptive & 44 & 40.7 & 10 & 34 & 14 & 23 \\
\hline Neuropathic & 13 & 12.0 & 8 & 5 & 2 & 9 \\
\hline \multicolumn{7}{|l|}{ Pain medications } \\
\hline Simple analgesics & 36 & 33.3 & 9 & 27 & 12 & 23 \\
\hline NSAIDs & 23 & 21.3 & 7 & 16 & 6 & 14 \\
\hline Anti-epileptics & 10 & 9.3 & 5 & 5 & 1 & 7 \\
\hline TCAs & 8 & 7.4 & 7 & 3 & 1 & 5 \\
\hline Tramadol & 6 & 5.6 & 3 & 3 & 0 & 5 \\
\hline Opiates & 44 & 40.7 & 12 & 32 & 13 & 23 \\
\hline Intrathecal pump & 1 & 0.9 & 1 & 0 & 0 & 0 \\
\hline
\end{tabular}

Abbreviation: NSAIDs, non-steroidal anti-inflammatory drugs; SCI, spinal cord injury; TCAs, tricyclic antidepressants.

with 0.16 (IQR from -0.10 to 0.45 ) for the secondary tumour group. Furthermore, the incomplete SCI group had significantly better $(P=0.003)$ FIM motor efficiencies (0.33, IQR 0.14-0.65) compared with complete lesions (0.08, IQR 0.0-0.33).

\section{Pain}

Pain was present in $52 \%$ of patients and pain that adversely impacted on rehabilitation was documented in $21 \%$ of cases, with the majority involving patients with secondary tumours and due to nociceptive pain (Table 3$)$. Pain had a significant $(P=0.01)$ adverse influence on the rehabilitation process, with FIM motor efficiencies of 0.09 in those with pain documented as being present compared with 0.39 in those without pain. Patients without pain demonstrated greater FIM motor efficiencies in both the primary and secondary tumour groups, with greater FIM motor efficiencies noted in the primary tumour groups without pain ( 0.57 vs 0.29$)$. Patients with pain also had a significantly longer $(P=0.03)$ median LOS of 56 days (IQR 32.5-84.5) compared with patients without pain, who had a median LOS of 42 days (IQR 20.5-72.5). Significantly lower motor discharge FIM scores were observed in both primary and secondary tumour groups with pain (Table 4). The presence of pain conferred a 30\% reduction in the median FIM motor discharge scores in the primary tumour group, and a $56 \%$ reduction in the secondary tumour group.

\section{Quality audit}

A total of 10 medical records were randomly checked for data integrity and all data items were validated to be correct.

\section{DISCUSSION}

This study has shown that patients with primary and secondary tumours benefit from specialist inpatient SCI rehabilitation. Patients with a primary tumour showed greater FIM motor gains and FIM motor efficiencies, despite having a longer median LOS. The presence of troublesome pain had a significant adverse impact on functional gains and LOS.

Most studies to date of SCI due to tumour have concentrated on prognostication and functional outcome without examining the effect of pain. Previous studies have usually reported total FIM scores, ${ }^{10}$ with only a few reporting separate motor and cognitive subscales. Our results differ from previous studies that reported mean FIM motor gains of $32^{\text {(ref. 21) }}$ and $15^{\text {(ref. 13) }}$ and are unique in that they reported separate results for patients with primary and secondary tumours. It would appear from our results that patients with secondary tumours causing SCI have more problems affecting their outcome and progress in rehabilitation than patients with a primary tumour. Pain is one of these problems, but there may be others that were not formally studied in this project.

Pain is generally a common sequelae post SCI. In a case series of traumatic SCI patients, $92 \%$ of patients on admission required treatment for pain. ${ }^{22}$ Another study in a cancer rehabilitation setting 
Table 4 Raw discharge motor FIM by tumor type and pain

Primary

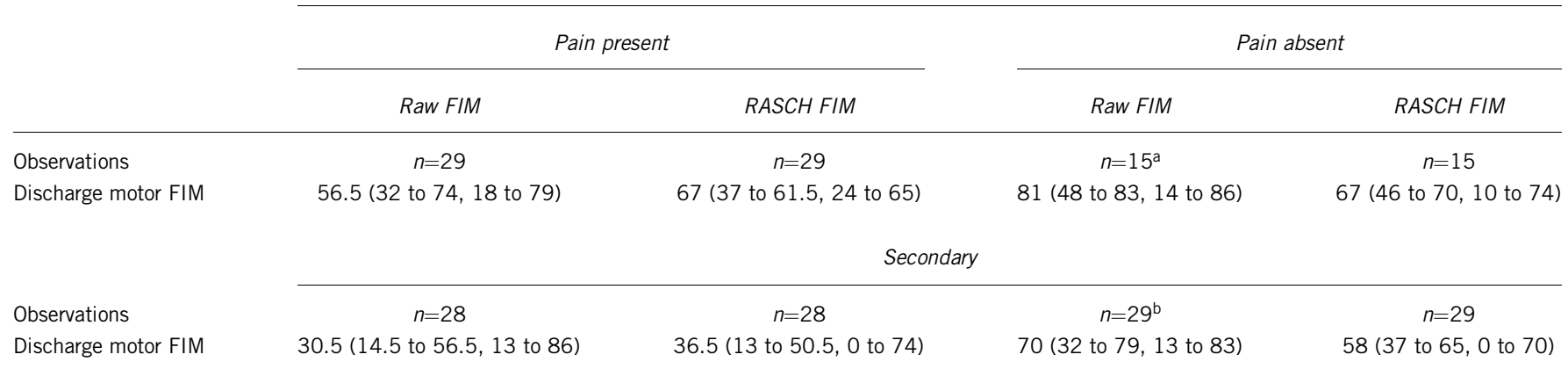

Abbreviations: FIM, Functional independent measure; IQR, interquartile range.

FIM scores listed as median (IQR, range).

a $P=0.008$ by Mann-Whitney test.

${ }^{\mathrm{b}} P=0.004$ by Mann-Whitney test.

reported that SCI rehabilitation reduces pain and depression compared with controls. ${ }^{14}$ The influence of pain on FIM motor efficiencies reported here was not surprising and to our knowledge has not previously been quantified.

It is important that the prognosis should be considered when assessing a patient with a SCI due to tumour for rehabilitation. Factors such as the patient's performance status and visceral metastases can be useful when attempting to prognosticate functional outcome for patients. ${ }^{23}$ Knowledge of the prognosis is crucial during the assessment process, as those not appropriate for a specialist SCI rehabilitation programme can be redirected to more appropriate settings. This needs to be tempered, however, with the limited alternative options besides palliative care.

Given the varying survival of patients with SCI due to tumour, ${ }^{10,12}$ rehabilitation teams must balance the goals and prognosis with an appropriate LOS to optimise time for patients to be out of hospital. This group of patients has unique issues that separate them from other causes of SCI. Consequently, the rehabilitation team needs to modify their therapy paradigm and inpatient goals. These patients may not be able to participate as fully as other patients, they may deteriorate and need transfer back to an acute hospital, ${ }^{24}$ a palliative care unit, or may die. Close and effective communication between the rehabilitation, oncology and palliative care teams are crucial to optimising patient outcomes, especially pain management. On the other hand, in those patients with a better prognosis, it is also important to plan post discharge rehabilitation in order to continue therapy for functional goals, if appropriate. Patients should also have periodic post discharge SCI medical rehabilitation reviews. These are to monitor and treat relevant SCI issues and advise the patient, general practitioner and other medical specialists of relevant management options.

Given the number of patients admitted over the study period, it would be very difficult to prospectively recruit larger numbers of patients with SCI due to tumour in a rehabilitation population within a shorter timeframe, without major resources and cooperation from multiple centres.

A limitation of this project includes the various weaknesses associated with any retrospective study. Inaccuracies from incorrect documentation may exist and there were problems with missing FIM scores. This study also reflects the experience of a single unit.

The information regarding pain must be interpreted somewhat cautiously. The definition of pain was limited by the retrospective nature of this study and the absence of any measure of pain intensity.
The presence of pain was based on medical file documentation and medication prescription. The main concern we have is the likelihood that our approach under-estimated the true prevalence. Our prevalence of $52 \%$ is lower when compared with other reports in the SCI literature. 22,25 Prospective information to characterise the study population further, such as with the use of formal psychological and pain assessments, would add weight to our findings. Future prospective studies may examine this.

Further research is needed into rehabilitation for patients with SCI from tumour. Areas for focus include the selection criteria and team processes to optimise a short inpatient stay balancing functional improvement and reduction in the burden of care and the prevalence, treatment and impact of pain.

\section{CONFLICT OF INTEREST}

The authors declare no conflict of interest.

1 Barron KD, Hirano A, Araki S, Terry RD. Experiences with metastatic neoplasms involving the spinal cord. Neurology 1959; 9: 91-106.

2 Prasad D, Schiff D. Malignant spinal-cord compression. Lancet Oncol 2005; 6: $15-24$.

3 Loblaw DA, Laperriere NJ, Mackillop WJ. A population-based study of malignant spinal cord compression in Ontario. Clin Oncol (R Coll Radiol) 2003; 15: 211-217.

4 Schellinger KA, Propp JM, Villano JL, McCarthy BJ. Descriptive epidemiology of primary spinal cord tumors. J Neurooncol 2008; 87: 173-179.

5 McKinley WO, Seel RT, Hardman JT. Nontraumatic spinal cord injury: incidence, epidemiology, and functional outcome. Arch Phys Med Rehabil 1999; 80: 619-623.

6 New PW. Functional outcomes and disability after nontraumatic spinal cord injury rehabilitation: results from a retrospective study. Arch Phys Med Rehabil 2005; 86: 250-261.

7 Scivoletto G, Lapenna LM, Di Donna V, Laurenza L, Sterzi S, Foti C et al. Neoplastic myelopathies and traumatic spinal cord lesions: an Italian comparison of functional and neurological outcomes. Spinal Cord 2011; 49: 799-805.

8 Eriks IE, Angenot EL, Lankhorst GJ. Epidural metastatic spinal cord compression: functional outcome and survival after inpatient rehabilitation. Spinal Cord 2004; 42. 235-239.

9 McKinley WO, Conti-Wyneken AR, Vokac CW, Cifu DX. Rehabilitative functional outcome of patients with neoplastic spinal cord compressions. Arch Phys Med Rehabil 1996; 77: 892-895.

10 Parsch D, Mikut R, Abel R. Postacute management of patients with spinal cord injury due to metastatic tumour disease: survival and efficacy of rehabilitation. Spinal Cord 2003; 41: 205-210.

11 Guo Y, Young B, Palmer JL, Mun Y, Bruera E. Prognostic factors for survival in metastatic spinal cord compression: a retrospective study in a rehabilitation setting. Am J Phys Med Rehabil 2003; 82: 665-668.

12 Tan M, New P. Survival after rehabilitation for spinal cord injury due to tumor: a 12-year retrospective study. J Neurooncol 2011; 104: 233-238. 
13 McKinley WO, Huang ME, Brunsvold KT. Neoplastic versus traumatic spinal cord injury: an outcome comparison after inpatient rehabilitation. Arch Phys Med Rehabil 1999; 80: 1253-1257.

14 Ruff RL, Ruff SS, Wang X. Persistent benefits of rehabilitation on pain and life quality for nonambulatory patients with spinal epidural metastasis. J Rehabil Res Dev 2007; 44: 271-278.

15 National Centre for Classification in Health. The International Statistical Classification of Diseases and Related Health Problems, 10th Revision, Australian Modification (ICD-10-AM), 2nd edn. National Centre for Classification in Health, Sydney, 2000.

16 Marino RJ, Barros T, Biering-Sorensen F, Burns SP, Donovan WH, Graves DE et al. International standards for neurological classification of spinal cord injury. J Spinal Cord Med 2003; 26(Suppl 1): S50-S56.

17 Guide for the Uniform Data Set for Medical Rehabilitation (including the FIM(TM) instrument). Version 5.1. State University of New York: Buffalo, NY, USA, 1997.

18 Heinemann AW, Linacre JM, Wright BD, Hamilton BB, Granger C. Relationships between impairment and physical disability as measured by the functional independence measure. Arch Phys Med Rehabil 1993; 74: 566-573.
19 New PW, Simmonds F, Stevermuer T. A population-based study comparing traumatic spinal cord injury and non-traumatic spinal cord injury using a national rehabilitation database. Spinal Cord 2011; 49: 397-403.

20 Heinemann AW, Hamilton BB, Granger CV, Wright BD. Final Report: Rating Scale Analysis of Functional Assessment Measures. Rehabilitaiton Institute of Chicago: Chicago, IL, USA, 1991.

21 Tooth L, McKenna K, Geraghty T. Rehabilitation outcomes in traumatic spinal cord injury in Australia: functional status, length of stay and discharge setting. Spinal Cord 2003; 41: 220-230.

22 Donnelly C, Eng JJ. Pain following spinal cord injury: the impact on community reintegration. Spinal Cord 2005; 43: 278-282.

23 Rades D, Rudat V, Veninga T, Stalpers LJ, Hoskin PJ, Schild SE. Prognostic factors for functional outcome and survival after reirradiation for in-field recurrences of metastatic spinal cord compression. Cancer 2008; 113: 1090-1096.

24 Alam E, Wilson R, Vargo M. Inpatient cancer rehabilitation: a retrospective comparison of transfer back to acute care between patients with neoplasm and other rehabilitation patients. Arch Phys Med Rehabil 2008; 89: 1284-1289.

25 Werhagen L, Hultling C, Molander C. The prevalence of neuropathic pain after nontraumatic spinal cord lesion. Spinal Cord 2007; 45: 609-615. 